\title{
Individual variation in the ability of Columbian ground squirrels to select an optimal diet
}

\author{
MARK E. RITCHIE \\ School of Natural Resources, University of Michigan, Ann Arbor, MI 48109-1115, USA
}

\begin{abstract}
Summary
Columbian ground squirrels (Spermophilus columbianus) were examined for ability to select a diet that maximizes daily energy intake (optimal diet) under free-living field conditions. The optimal diet for each squirrel was determined given constraints (e.g. body size, feeding time) on individual foraging behavior. Most squirrels $(63 \%)$ consumed a diet not significantly different from one that would maximize their daily energy intake. The remainder $(37 \%)$ approached an energy maximized diet but appeared to make some incorrect foraging decisions. Both males and females appeared to approach energy maximized diets. An individual's deviation from its optimal diet is relatively constant within a season and not significantly affected by immediate environmental influences such as food abundance, thermal conditions and social environment. The energy cost of deviating from an optimal diet may be large enough to affect fitness. These results suggest that the ability to select an optimal diet can be viewed as a behavioral trait that might be subject to natural selection.
\end{abstract}

Keywords: Optimal foraging; individual variation; ground squirrels; energy budget; ontogeny.

\section{Introduction}

The idea that an animal should choose the diet that maximizes its fitness (survival and production), or forage optimally, was developed by MacArthur and Pianka (1966), Emlen (1966), Schoener (1971), Pulliam (1974), and Charnov (1976). These studies suggested that an animal should forage optimally, or maximize its net intake of some 'currency' (e.g. energy/ nutrient or time). Many subsequent studies have examined these ideas by testing whether or not animals choose the diet that maximizes their net rate of energy intake (for reviews see Pyke et al., 1977; Pyke, 1984; Stephens and Krebs, 1986). Most of these studies compute the optimal diet for an average individual in a population and compare this value against the observed average diet (e.g. Werner and Hall, 1974; Krebs et al., 1974, 1977; Belovsky; 1978). In most cases, observed diets agree fairly well with predicted optimal diets (Stephens and Krebs, 1986).

These foraging studies generally have not examined whether individual animals can forage optimally. Krebs et al. (1977), Houston et al. (1980) and Rechten et al. (1983) suggested that some individuals may approach an optimal diet more closely than others but their small sample sizes prevented a detailed examination. Arnold $(1977,1981)$ found variability in the food preference of individual garter snakes (Thamnophis sp.) but he did not test whether individuals foraged optimally.

Most studies of optimal foraging have implicitly assumed that individuals in a population approach a single optimal diet. This view may be incorrect, since potential constraints on diet choice, such as available feeding time or nutrient requirements (Westoby, 1974; Pulliam, 1975; 
Belovsky, 1978), may differ among individuals. Consequently, it may be unrealistic to ask whether individuals can achieve a single optimal diet. A more appropriate question might be whether an individual can select an optimal diet, given its morphology, physiology, and immediate foraging environment.

To answer this question, it is important to establish what determines an individual's optimal diet. Depending on the 'currency' that is important for fitness (energy, nutrients, time, etc.), individuals might adopt one of two alternative foraging strategies (Schoener, 1971; Belovsky, 1978; Hixon, 1982): energy maximization or time minimization. Energy maximizers acquire the greatest energy/nutrient within their available feeding time. This strategy is expected when energy or nutrients are most important for an individual's fitness. On the other hand, time minimizers satisfy their minimum energy/nutrient requirements in the least time. This strategy is expected when time (e.g. time spent searching for mates or avoiding predators) is the most important currency for fitness. Individuals within the same population may differ in their particular foraging strategy, depending on the activities that maximize their fitness. For example, males might be time minimizers because it might be more important to search for mates than to forage. On the other hand, females might be energy maximizers because their fitness might be determined by the amount of energy/nutrients they can invest in offspring.

I attempted to determine if individual Columbian ground squirrels (Spermophilus columbianus) varied in their ability to select their individual optimal diet. This involved two steps: (1) determining the optimal diet for each individual, and (2) determining if some individuals deviate from their optimal diet. I measured the foraging constraints of individually marked squirrels. I then calculated an optimal diet for each individual, using a linear programming optimization model (Belovsky, 1978) constructed with the foraging constraints determined for each individual. The predicted optimal diet was then compared with the individual's observed diet, which was measured from fecal samples. The difference between an individual's predicted optimal diet and its observed diet (deviation from an optimal diet) was used as a measure of its foraging performance.

If individuals vary in how closely they approach their optimal diet, this variation might be caused by differences among individuals in their foraging strategies. While the immediate foraging environment (e.g. food abundance, thermal conditions, social environment) undoubtedly will affect an individual's foraging constraints, it may also affect an individual's ability to make foraging decisions. In addition, individuals might also vary in their deviation over time. I therefore determined whether: (1) sex-age classes differ in their foraging strategy as reflected by an individual's ability to approach their optimal diet; (2) an individual's ability to select an optimal diet is affected by its immediate foraging environment; and (3) an individual's deviation changes over time within a season.

If these factors are not found to be important, then deviation from an optimal diet is essentially independent of the time or environment in which the behavior is measured. If so, an individual's deviation can potentially be viewed as a trait, similar to other morphological or physiological traits, such as body size or assimilation efficiency (Endler, 1986; Arnold and Wade, 1984a, b).

\section{Methods}

Study sites

I conducted the study from May to July over four years, 1983-6, on open, grazed pastures three kilometers apart near the National Bison Range, Montana (Sites JB and RJ-2 in Belovsky and Ritchie, submitted). Columbian ground squirrels are burrowing sciurid rodents that are active at the study sites from early April until mid-July. They spend the remainder of the year hibernating/ estivating (Shaw, 1925; Manville, 1959). 
At the study sites, ground squirrels eat primarily two food types: monocotyledonous plants (monocots) which are mostly grasses Poa pratensis, Dactylis glomerata, Bromus tectorum, Festuca pratensis and Agropyron repens); and dicotyledonous plants (diocots) which are mostly herbs Taraxacum sp., Erodium cicutarium, Polygonum aviculare, Medicago sativa and Cirsium foliosum). Monocots represent 55-70\% of the dry biomass of vegetation at the study sites, and dicots the remaining $30-45 \%$. The two sites did not differ significantly in plant biomass, species composition, or thermal environment (Belovsky and Ritchie, 1989).

\section{Experimental design}

To determine if foraging behavior varied among individuals, I observed the foraging behavior of each of 109 marked free-living individuals (approximately 27 each year of the study) in the field. The parameters necessary for construction of a linear programming optimization model for each individual were measured (see later). Squirrels were live-trapped, weighed, sexed, and individually marked with metal ear tags and colored ear streamers made from Safe-Flag material. These markings are visible through binoculars at distances greater than $100 \mathrm{~m}$. Trapping occurred in May just before and after juveniles were weaned. Individuals weighing less than $300 \mathrm{~g}$ were considered to be juveniles, those weighing 300-500 g were considered to be yearlings, and those weighing greater than $500 \mathrm{~g}$ were considered to be adults (Boag and Murie, 1981a).

To determine if foraging behavior was influenced by environmental factors, I compared the foraging behavior of 22 of the 109 individuals under two conditions: (1) in the field where social interactions and home range quality might affect foraging; and (2) isolated in $5 \times 5 \mathrm{~m}$ vegetated enclosures, where food was much more abundant and social influences were greatly reduced compared to field conditions for all individuals tested. Two chicken-wire enclosures were built side-by-side to allow two individuals to be tested at one time. Hay bales were stacked between enclosures to prevent squirreis from interacting. Squirrels were allowed to acclimate and learn the distribution of vegetation for two days. On the third day, observed diets and the parameters of a linear programming model were measured, as described later for free-living individuals. Each of the 22 individuals was tested once. These tests were conducted approximately $4-6$ weeks after individuals had been observed in the field.

For individuals tested during the same season, no fallow period was allowed between consecutive trials. As a result, vegetation was gradually depleted in the enclosures over the season. To compensate for this depletion, enclosures were irrigated with the equivalent $7 \mathrm{~cm}$ of precipitation per week, eight times the normal rate for June at my study site. This ensured that: (1) the biomass of vegetation within the enclosures would be at least four times that found at the field study sites; and (2) dicots would also have higher relative abundance in the enclosures ( $45 \%$ monocots, $55 \%$ dicots) than at the field study sites.

The enclosures also differed from the field study sites in available feeding time. The average daily high temperature during the enclosure experiments was $4^{\circ} \mathrm{C}$ higher than earlier in the season, and this reflected increased heat stress on ground squirrels active during the day. As a result, individuals had an average of $1.5 \mathrm{~h}$ less feeding time in the enclosures.

Due to the difference in food abundance and thermal environment, the enclosure and field foraging environments are very different, although the enclosure conditions were within the range found at other ground squirrel colonies (Belovsky and Ritchie, 1989; Webb, 1980). Consequently, the impact of the immediate foraging environment can be determined by comparing an individual's foraging behavior in these two environments. The conditions within the enclosures changed between trials, but these differences were small compared to that between the enclosures and the field. Furthermore, the constraints for each individual were measured according to the enclosure conditions experienced by each individual. The $4-6$ week 
time difference in measuring foraging behavior also allows the consistency of foraging behavior within a season to be tested.

\section{Model parameters: methods and assumptions}

To predict the optimal diet for each of the 109 individuals, I constructed a linear programming model for each individual (Westoby, 1974; Belovsky, 1978). Linear programming is the most appropriate optimization technique for this situation for two reasons (Belovsky, 1978, 1984). First, monocots and dicots are patchily distributed relative to each other, since their abundance at the study sites was negatively associated (Belovsky, 1986). Consuming one food type, therefore, precludes consumption of the other. Second, linear programming allows constraints on foraging behavior (e.g. digestive capacity, available feeding time) to be considered.

Each model predicts the amount of the two food types, monocots or dicots, consumed by an individual, subject to three constraints: (1) the individual's maximum available feeding time (activity time) and its utilization by the cropping rate of plant foods; (2) the individual's maximum daily digestive capacity and its utilization by food bulk; and (3) the individual's minimum daily energy requirement and its satisfaction by the digestible energy content of plant foods. These constraints can be written in linear form as:

$$
C \leq a m+b d
$$

or

$$
C \geq a m+b d
$$

where $C$ is the constraint value and $a$ and $b$ convert the amount (dry wt) of monocot $(m)$ and dicot $(d)$, respectively, into the units of the constraint. The constraint equations define the boundaries of a set of feasible diets which satisfy daily energy requirements but do not exceed maximum daily feeding time or digestive capacity (Belovsky, 1978).

Previous work suggests that the feeding time, digestive capacity, and energy constraints are the only constraints that are important for ground squirrels at my study site (G. E. Belovsky and M. E. Ritchie, submitted). For further justification of linear programming and the particular constraints used in this study, see Belovsky $(1984,1986)$ and G. E. Belovsky and M. E. Ritchie (submitted).

The linear programming model can be solved for the proportions of monocot and dicot composing an energy maximized and time minimized diet (Fig. 1). The observed diet of an individual can then be compared with these predicted diets to determine an individual's deviation from its optimal diet.

The parameters in each constraint equation were measured for each individual. The collection techniques used to measure each parameter are described below.

Feeding time. To determine an individual's energy maximized diet, the maximum time available for feeding must be estimated. Maximum available feeding time for an individual can be estimated from the amount of time the individual was active each day. An individual was considered active if it was above-ground and not at a burrow entrance (Belovsky and Slade, 1986). Activity time is likely to represent maximum available feeding time for ground squirrels because it appears to be limited by the thermal environment (Belovsky, 1986; Belovsky and Ritchie, submitted).

Activity time and cropping rates were measured in the field for each individual. Observations were begun no later than six days after the first individual was captured at each colony to ensure correspondence between foraging observations and observed diets. The colony was scanned 


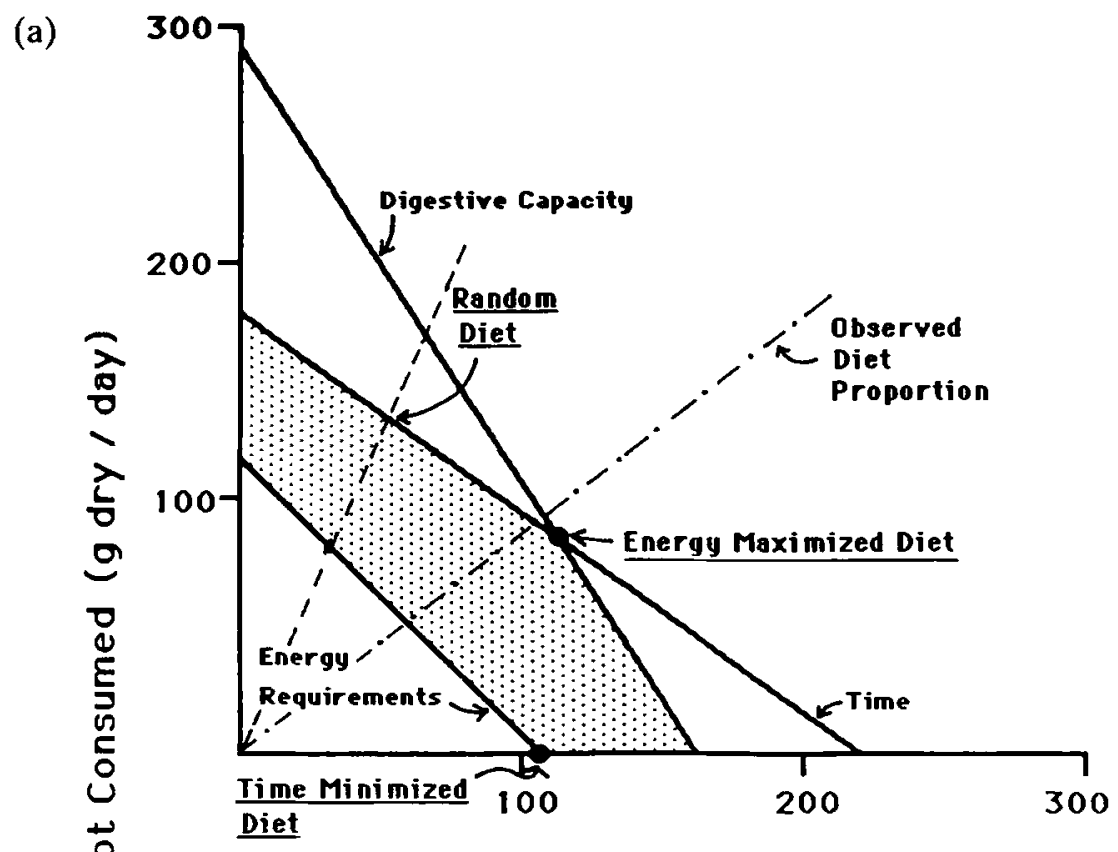

(b)

응

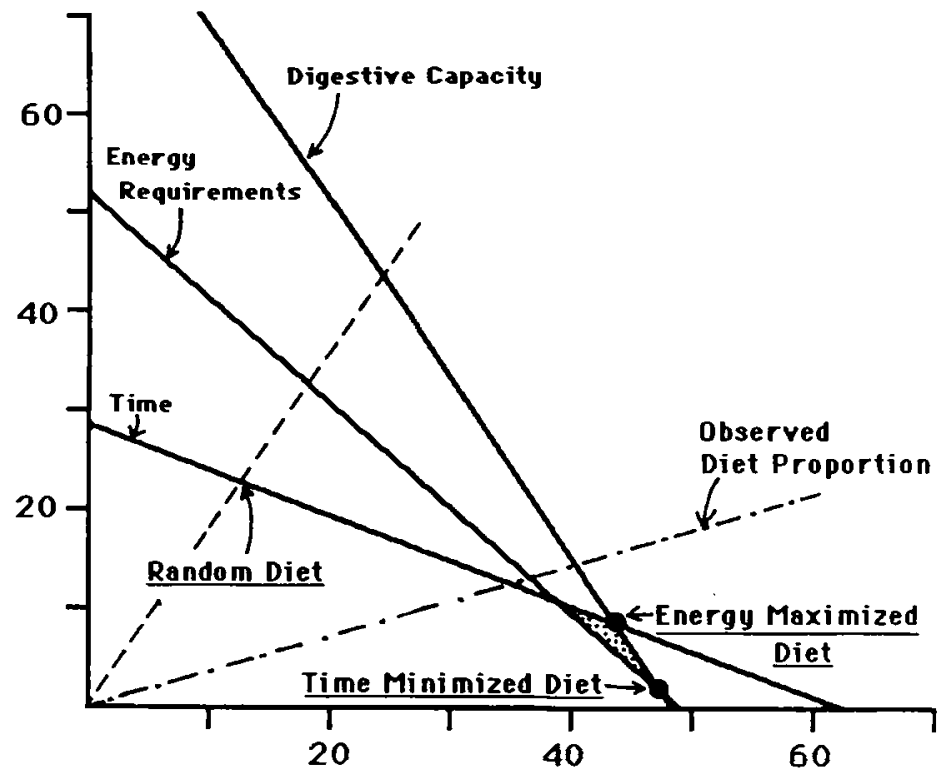

Dicot Consumed (g dry / day)

Figure 1. Examples of the linear programming models for two individuals. (a) The model for an adult female in which the time minimized diet is $100 \%$ dicots (Case (1) in text). (b) The model for a juvenile female in which the time minimized diet is a mixture of monocots and dicots (Case (2) in text). Constraints are labelled and the region of feasible diets is shaded. Equations and parameters for the constraint lines are shown in Tables 2 and 3. The two optimal solutions, energy maximization and time minimization, are indicated by closed circles. The random diet proportion is indicated by the alternately dashed line. 
every 15 minutes throughout the day for at least three days. During each scan, the activity of each individual was recorded. Activity time was computed as the number of 15 minute scan intervals an individual was observed active (Belovsky and Slade, 1986). The mean activity time for the three-day period was used in the linear programming model.

Cropping rates for each individual were measured separately for monocots and dicots as the product of the average number of bites taken per minute and the mean dry weight of bites. Biting rates were measured by counting the number of bites of a single food type taken by an individual in one minute at close range $(<20 \mathrm{~m})$. These rates were then averaged to obtain a single mean for an individual.

Ground squirrels at my site normally take one item per bite. Mean dry weight of these bites was estimated by measuring, with calipers, the diameter (stem) or width (grass blade) of the remaining stub of a plant which had recently been fed upon by a ground squirrel. Using the calipers, I located the point which matched the diameter of the stub tip along the stem/blade of the nearest similar plant. The portion of the next nearest plant above the matched diameter was clipped, dried and weighed. Using this systematic criterion for selecting 'matched' bites accounts for the possibility that individuals might eat plant parts that had previously been eaten by either ground squirrels or cattle, and avoids the potential for overestimating bite size by selecting only plant parts which had never been eaten.

Bite sizes (five of each food type) were estimated for each of 15 individuals during the first year of the study (Table 1 ). Average bite size was significantly different between adults $(n=7)$ and juveniles $(n=8)$ but was not different among individuals within an age class (see Results). In subsequent years, I therefore collected 20 matched bites of both monocots and dicots to represent the average for each age class, at each site, and during each year of the study.

Table 1. A comparison of average bite sizes among individuals within and between age classes.

\begin{tabular}{|c|c|c|}
\hline \multirow[b]{2}{*}{ Individual } & \multicolumn{2}{|c|}{ Bite size ( $\mathrm{mg} \pm$ S.D., $n=5)$} \\
\hline & Monocot & Dicot \\
\hline \multicolumn{3}{|l|}{ Juveniles } \\
\hline 1 & $18 \pm 6$ & $45 \pm 8$ \\
\hline 4 & $21 \pm 8$ & $44 \pm 9$ \\
\hline A & $20 \pm 6$ & $38 \pm 13$ \\
\hline C & $21 \pm 8$ & $43 \pm 7$ \\
\hline D & $24 \pm 8$ & $42 \pm 10$ \\
\hline E & $23 \pm 7$ & $37 \pm 7$ \\
\hline $\mathbf{F}$ & $24 \pm 10$ & $47 \pm 11$ \\
\hline $\mathrm{G}$ & $18 \pm 8$ & $43 \pm 11$ \\
\hline Average & $25 \pm 9$ & $43 \pm 21$ \\
\hline \multicolumn{3}{|l|}{ Adults } \\
\hline A & $30 \pm 14$ & $55 \pm 23$ \\
\hline $\mathbf{B}$ & $34 \pm 16$ & $57 \pm 21$ \\
\hline $\mathrm{C}$ & $30 \pm 11$ & $59 \pm 19$ \\
\hline $\mathrm{D}$ & $32 \pm 14$ & $60 \pm 24$ \\
\hline 1 & $35 \pm 13$ & $57 \pm 19$ \\
\hline 2 & $36 \pm 11$ & $62 \pm 24$ \\
\hline 3 & $31 \pm 16$ & $63 \pm 16$ \\
\hline Average & $32 \pm 15$ & $60 \pm 22$ \\
\hline
\end{tabular}


Digestive capacity. To predict the energy maximized diet, the maximum amount of food that can be processed in the digestive tract must also be known. Daily digestive capacities for individuals were calculated as the product of the wet mass of the digestive organ (stomach + caecum) contents and the number of times per day that food passed through the digestive tract (turnover rate). At one site not used in this study, 48 ground squirrels were shot after their morning feeding period and the contents of their stomach and caecum were weighed. The 48 individuals were separated into eight body size classes. Within each size class, the individual with the maximum digestive organ mass was determined. Using these eight individuals, digestive organ capacity was regressed against body size. Further details are provided in Belovsky and Ritchie (1989). The regression equation obtained from this analysis was used to estimate digestive organ capacity for each individual in the study, given its body mass (see Results).

Turnover rate was measured as the number of times per day the stomach could fill and empty. Ten captive squirrels of a range of body sizes $(220-550 \mathrm{~g})$ were provided with one 'meal' (digestive organ contents) of chopped alfalfa (Medicago sativa), a common food at my study site. Short strands of indigestible cotton thread were added to the food as a label. Turnover time for one 'meal' was estimated as the mean time individual threads were retained in the digestive tract.

Ground squirrels are diurnal and, in summer, are active during a $12 \mathrm{~h}$ period (Belovsky and Slade, 1986; Belovsky, 1986; Belovsky and Ritchie, 1989). I assumed that they could digest food for $12 \mathrm{~h}$ per day and enter the nocturnal inactive period (also $12 \mathrm{~h}$ ) with a full digestive organ. Given that turnover time for one 'meal' was much less than $12 \mathrm{~h}$, I assumed the digestive tract was empty by the next morning. Turnover rate, therefore, was calculated as the number of 'meals' which could pass through the digestive tract in a day: $(12 \mathrm{~h} /$ turnover time $)+1$. Turnover rates decrease significantly with body size (Fig. 2) (see Results), so a regression equation of turnover rate as a function of body size was used to calculate turnover rate for each individual. Food bulk (wet wt/dry wt) values for monocots and dicots were obtained from Belovsky (1986) and were assumed to be the same for all individuals.

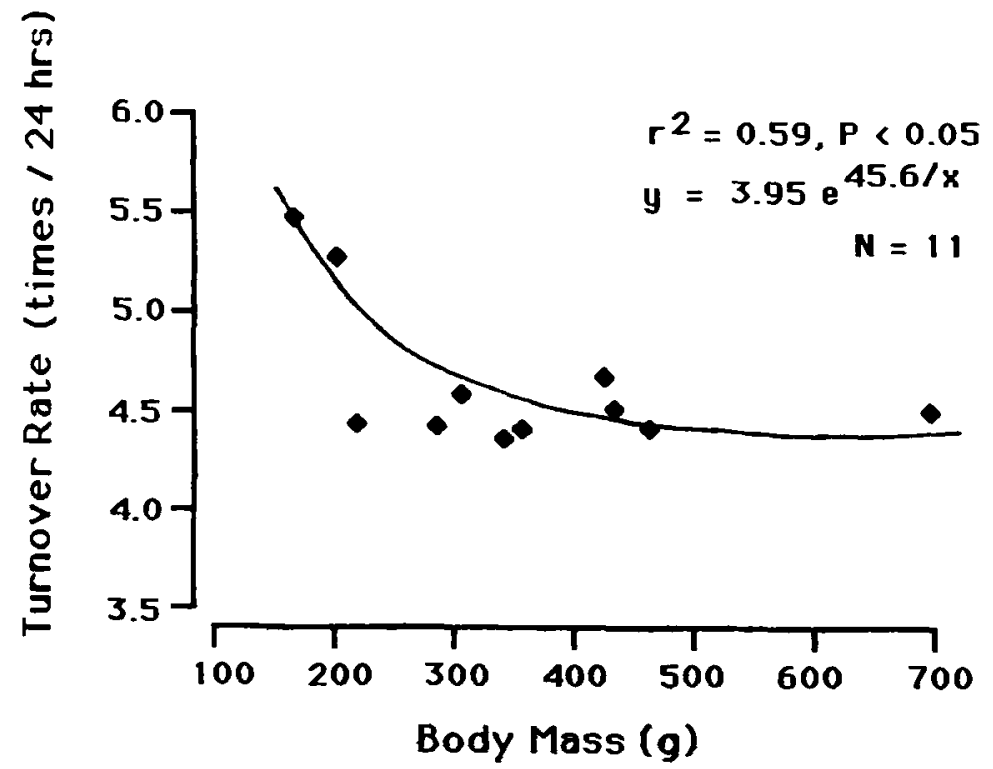

Figure 2. The relationship between turnover rate (number of full digestive organs which can be passed . through the digestive tract in $24 \mathrm{~h}$ ) and body size for 11 individuals. The non-linear function was fitted to the data using a log-inverse transformation: $\ln y=a(1 / x)+b$. This model provided the best fit to the data. 
Daily energy requirement. To determine a time minimized diet, the daily energy requirement must be known. For ground squirrels, the average daily energy requirement is the average daily active metabolism plus the average daily energy intake needed to store sufficient fat for hibernation. Energy requirements were estimated as a multiple of basal metabolic rate (BMR) for each of the 109 individuals in the study. BMR (kJ/day) was calculated on the basis of each individual's body size using Kleiber's (1961) estimate: BMR $=239 W^{0.75}$ ( $W$ in $\mathrm{kg}$ ). BMR was then multiplied by a constant to estimate the average daily energetic requirement (see Results). This constant reflects the estimated cost of activity and hibernation, as determined from previous studies of ground squirrel metabolism (see Results). The digestible energy content of each food type was obtained from Belovsky (1986) and assumed to be the same for all individuals. This assumption is verified to a limited extent in Ritchie (submitted).

Observed diets. The proportion of monocots and dicots consumed by each of the 109 individuals

Table 2. Parameters used in the linear programming models for two example individuals - (a) and (b) in Fig. 1.

\begin{tabular}{|c|c|c|}
\hline \multirow[b]{2}{*}{ Parameter } & \multicolumn{2}{|l|}{ Individual } \\
\hline & (a) & (b) \\
\hline Body size ( $W$ in $\mathrm{g}$ ) & 530 & 125 \\
\hline $\begin{array}{l}\text { Activity time }(T) \\
\text { (min/day, } n=\text { days) }\end{array}$ & $\begin{array}{l}570 \pm 16 \\
(3)\end{array}$ & $\begin{array}{l}225 \pm 21 \\
(3)\end{array}$ \\
\hline \multicolumn{3}{|l|}{$\begin{array}{l}\text { Cropping rates } \\
(\min / g \text { dry, } n=\min )\end{array}$} \\
\hline Monocots $\left(c_{\mathrm{m}}\right)$ & $\begin{array}{l}3.25 \pm 0.92 \\
(21)\end{array}$ & $\begin{array}{l}8.14 \pm 1.41 \\
(14)\end{array}$ \\
\hline Dicots $\left(c_{d}\right)$ & $\begin{array}{l}2.62 \pm 0.45 \\
(27)\end{array}$ & $\begin{array}{l}3.55 \pm 0.48 \\
(20)\end{array}$ \\
\hline $\begin{array}{l}\text { Digestive capacity }(D) \\
\text { (g wet mass/day) }\end{array}$ & $457.0 \pm 22.8$ & $125.6 \pm 6.3$ \\
\hline \multicolumn{3}{|l|}{$\begin{array}{l}\text { Food bulk } \\
\text { (wet mass/dry mass) }\end{array}$} \\
\hline $\begin{array}{l}\text { Monocots }\left(b_{\mathrm{m}}\right) \\
\text { Dicots }\left(b_{\mathrm{d}}\right)\end{array}$ & $\begin{array}{l}1.64 \pm 0.35 \\
2.67 \pm 0.75\end{array}$ & $\begin{array}{l}1.64 \pm 0.35 \\
2.67 \pm 0.75\end{array}$ \\
\hline $\begin{array}{l}\text { Energy requirements }(H) \\
(\mathrm{kJ} / \text { day) }\end{array}$ & 1029.5 & 494.6 \\
\hline \multicolumn{3}{|l|}{$\begin{array}{l}\text { Digestible energy content } \\
\text { (kJ/g dry) }\end{array}$} \\
\hline $\begin{array}{l}\text { Monocots }\left(e_{\mathrm{m}}\right) \\
\text { Dicots }\left(e_{\mathrm{d}}\right)\end{array}$ & $\begin{array}{l}9.44 \\
10.19\end{array}$ & $\begin{array}{l}9.44 \\
10.19\end{array}$ \\
\hline \multicolumn{3}{|l|}{$\begin{array}{l}\text { Proportion food in } \\
\text { environment ( } \% \text { biomass) }\end{array}$} \\
\hline $\begin{array}{l}\text { Monocots } \\
\text { Dicots }\end{array}$ & $\begin{array}{l}64.0 \\
36.0\end{array}$ & $\begin{array}{l}61.1 \\
38.9\end{array}$ \\
\hline Observed diet ( $\%$ Monocot) & $\begin{array}{l}43.4 \pm 4.1 \\
(5)\end{array}$ & $\begin{array}{l}26.7 \pm 3.9 \\
(5)\end{array}$ \\
\hline
\end{tabular}


was measured from five fecal pellets collected when an individual was captured. These five pellets were selected randomly from all pellets deposited by an individual during a single capture. Feces deposited by individuals varied widely in water content $(80-40 \%)$, suggesting that feces collected at capture represent food eaten over the previous $5 \mathrm{~h}$ of foraging (based on digestive turnover rate, see Results). Most individuals were captured only once before foraging observations, so five pellets from the most recent capture before foraging observations were used in the diet analysis for all 109 individuals. Because some individuals were captured more than once before foraging observations, diet variation among pellets within a day was compared to diet variation between days (see Discussion).

I used microhistological techniques to determine the diet proportion in each pellet (Storr, 1963; Sparks and Malechek, 1968; Hansen et al., 1977). These techniques have been widely used in determining herbivore diets, and their application to ground squirrel diets is detailed in Belovsky (1986) and Belovsky and Ritchie (submitted).

Variation in diet proportion among the five pellets collected within a day was used to estimate diet variability for each individual (e.g. in Table 2). The square root of the proportion of monocot in each pellet and in the predicted diet was Arcsin transformed. For each individual, I used $t$-tests to compare the mean ( \pm S.D.) diet of the five fecal pellets with the alternative optimal diets predicted by the linear programming model.

\section{Results}

\section{Model parameters and assumptions}

All of the linear programming model parameters were determined for each of the 109 individuals. As an example, the model parameters measured for two individuals are shown in Table 2 . The constraint equations used in the linear programming model are also presented (Table 3), showing

Table 3. Equations used to construct the linear programming models for each individual in the study. Variables in equations correspond to those in Table 2.

Food eaten

$m=$ amount of monocot (dry mass) consumed per day

$d=$ amount of dicot (dry mass) consumed per day

Time constraint

$$
T \geq c_{\mathrm{m}} m+c_{\mathrm{d}} d
$$

Digestive constraint

$$
\begin{aligned}
D & =\text { (turnover rate) } \times(\text { stomach }+ \text { caecum capacity }) \\
& =\left(3.95 \mathrm{e}^{45.6 / W}\right)(0.19 \mathrm{~W}) \\
& =0.75 \mathrm{~W} \mathrm{e}^{45.6 / W} \\
D & \geq b_{\mathrm{m}} m+b_{\mathrm{d}} d
\end{aligned}
$$

Hibernation energy requirement constraint

Adults:

$$
\begin{aligned}
& H=5.72 \times \mathrm{BMR}=5.72\left(1.63 W^{0.75}\right) \\
& =9.32 W^{0.75} \\
& H=8.12 \times \mathrm{BMR}=8.12\left(1.63 W^{0.75}\right) \\
& =13.23 W^{0.75} \\
& H \leq e_{\mathrm{m}} m+e_{\mathrm{d}} d
\end{aligned}
$$


how each parameter fits into the model. These parameters include maximum daily feeding time (activity time), cropping rates, maximum daily digestive capacity, food bulk, daily energy requirements, and digestible energy content of plants. Some of these parameters are extremely difficult to measure directly for each individual, so I assumed that they could be estimated from each individual's body size, or be treated as constant for all or part of the 109 individuals. Below, I present some justification for these assumptions.

The observed activity time of an individual was assumed to represent the maximum available feeding time, because activity time appears to be limited by the thermal environment. Using a heat flow model (Porter and Gates, 1969), Belovsky and Ritchie (1989) show that maximum activity time for ground squirrels might be set by the thermal environment. They successfully predict the level of activity each hour of the day for an average individual (predicted vs. observed, $r^{2}=0.76, n=12, p<0.001$ ). The predicted maximum feeding time for the average thermal conditions of late May to early June was $333 \mathrm{~min} /$ day and corresponded to the observed average activity time of $341 \mathrm{~min} /$ day. The average activity time for all individuals in this study was 328.0 ( $\pm 41.7 \mathrm{~s}$.D.) $\mathrm{min} /$ day, which is not significantly different from the predicted maximum $(t=$ 1.25 , $\mathrm{df}=108, p>0.20)$. As a result, observed activity times appeared to be limited by thermal conditions during the summer active season.

Limitations on the activity of an individual by the thermal environment depend on characteristics of the individual's home range (e.g. near tree shade, north vs. south facing slope, etc.) and the individual's ability to thermoregulate. Because these characteristics vary among individuals, some individuals had activity times above and below the predicted maximum for an average individual. As a result, activity time represents the only realistic estimate of maximum feeding time for an individual.

Bite sizes were assumed to be equal within an age class for a given site and year (Table 1). Bite size did not vary significantly among individuals within an age class for juveniles (Monocots: $F=$ 3.42, $\mathrm{df}=7,28, p=0.16$; Dicots: $F=4.06, p=0.095$ ) or adults (Monocots: $F=0.57, \mathrm{df}=6,24$, $p=0.63$; Dicots: $F=0.41, p=0.71$ ). The average bite size for all adults was significantly greater than that for all juveniles (Monocots: $F=6.17$, df $=2,39, p=0.021$; Dicots: $F=10.36, p=$ 0.004).

Daily digestive capacity was determined as the product of digestive organ capacity and daily turnover of food through the digestive tract. These two parameters were calculated for each of the 109 individuals on the basis of their body size, using regression equations of: (1) the maximum wet mass of the digestive organ contents $(K)$ vs. body size ( $W$ in $\mathrm{g})\left(r^{2}=0.99, n=8, p<0.01, K\right.$ $=0.19 W$; from Belovsky and Ritchie, 1989) and (2) turnover rate (T) vs. body size (Fig. 2) $\left(r^{2}=\right.$ $\left.0.59, n=11, p<0.01, T=3.02 \mathrm{e}^{45.6 / W}\right)$.

The average daily activity energy requirement for ground squirrels was estimated to be 2.93 \pm 0.8 (S.D.) $\times$ BMR, from the average of values presented in other studies of ground squirrels (Hudson and Deavers, 1973; Morhardt and Gates, 1974; Morhardt, 1975; Schitoskey and Woodmansee, 1978; Wang, 1979; Karasov, 1981). The average daily requirement for storing sufficient fat for hibernation can also be expressed as a multiple of BMR (Ritchie, submitted): $2.74 \times$ BMR for adults and yearlings and 5.26 $\times$ BMR for juveniles. These estimates are based on the annual activity cycle at my study site (Belovsky and Ritchie, 1989), an estimate for the proportional reduction in basal metabolism during hibernation (Wang, 1979), and an estimate of the efficiency of conversion of stored fat into metabolizable energy (Kilgore and Armitage, 1978). The requirement is larger for juveniles because they have fewer days to store fat than adults. Therefore, the estimated total daily energy requirement for maintenance and successful hibernation/estivation was 5.67 ( \pm 1.13s.D.) $\times$ BMR for adults and $8.19( \pm 1.64) \times \mathrm{BMR}$ for juveniles. 


\section{Model solutions}

According to the linear programming algorithm, solutions for optimal diets (with two food types) occur at the intersection of two constraints or at the intersection of a constraint and an axis (Intriligator, 1971). The animals in this particular study are limited in their intake of dicots by digestive capacity because dicots are bulkier than monocots. On the other hand, individuals are limited in their intake of monocots by activity time because monocots are cropped more slowly than dicots (Fig. 1, Table 2). This implies a trade-off between filling the digestive organ and utilizing maximum feeding time. For each individual in this study, the energy maximized solution occurs at the intersection of the activity time and digestive constraints.

The time minimized solution depends on the magnitude of the energy requirement relative to the other two constraints. In this study, time minimized diets occurred at one of two solutions: (1) the intersection of the energy requirement constraint and the dicot axis (93 individuals, both adults and juveniles); and (2) the intersection of the energy requirement and digestive capacity constraints (16 individuals, all juveniles). Case (1) predicts a diet of $100 \%$ dicots. Case (2) predicts a mixed diet that includes a proportion of monocots between zero and

(a)

Figure 3. The correlation between predicted energy maximized diets and observed diets (as $\%$ monocot in the diet) is presented for (a) all 109 individuals in the field, and (b) 22 individuals in the enclosures. In (a), closed circles represent adults and closed squares juveniles. In both figures, the diagonal line represents perfect agreement.

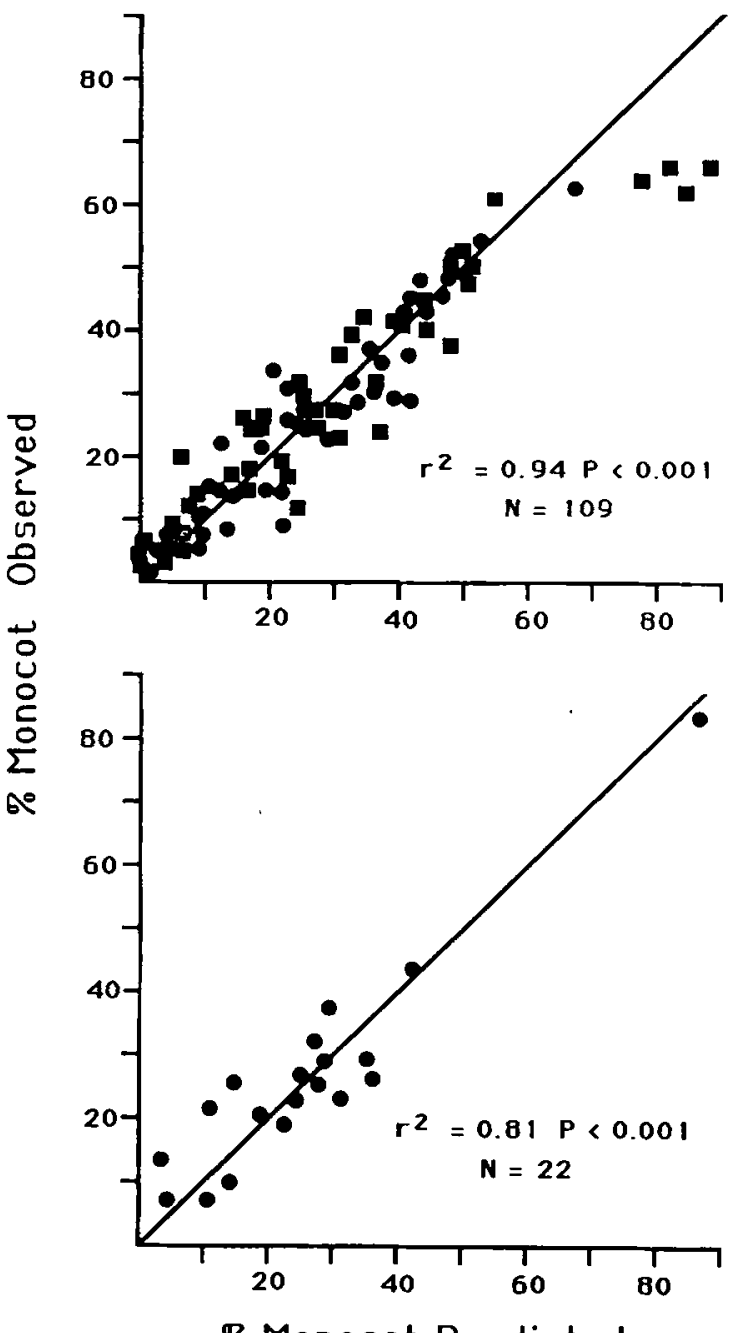

$\%$ Monocot Predicted 
that in the energy maximized diet. In all cases, the energy maximized and time minimized diets were distinguishable.

I also considered that ground squirrels might forage randomly, i.e. eat plant foods as they encounter them. Squirrels consuming a random diet would be expected to eat the two food types in their proportion (by dry biomass) in the environment (Belovsky and Ritchie, 1989) without exceeding digestive capacity or feeding time, whichever is limiting for that diet proportion.

Solutions of the linear programming model are presented for the two example individuals (Fig. $1 \mathrm{a}, \mathrm{b}$ ), using the parameter measurements in Table 2. These two examples show Cases (1) and (2) for the time minimized solution.

Individuals appear to approach energy maximized diet proportions but not time minimized or random diet proportions. Predicted energy maximized diets are strongly correlated with observed diets (Fig. 3) $\left(r^{2}=0.94, n=109, p<0.001\right)$ but predicted time minimized and random diets are not $\left(r^{2}=0.02, p>0.20 ; r^{2}=0.03, p>0.20\right.$; respectively). Individuals also appear to consume the amount of food predicted for energy maximizers. Captive individuals of various body sizes $(220-550 \mathrm{~g})$ were fed a mixture of monocots and dicots, and ate a similar amount of food (dry mass) to that predicted by the linear programming model constructed from observations of their foraging behavior in the field $\left(r^{2}=0.80, p<0.001, n=15\right)$.

I also attempted to determine if the immediate foraging environment influenced an individual's foraging behaviour. Observed diets are strongly correlated with predicted energy maximized diets for the 22 individuals tested (body size range: $260-700 \mathrm{~g}$ ) in the enclosure environments (Fig. 3b) $\left(r^{2}=0.81, p<0.01\right)$. Individuals, therefore, appear to approach their energy maximized diets in the enclosures.

\section{Deviation from an optimal diet}

For each individual, deviation from its energy maximized diet was used as a measure of its foraging performance. Deviation from an optimal diet is proportional to the number of foraging 'mistakes' made by an individual. Hence, the greater the deviation in diet proportion, the greater the number of mistakes. Deviation from an optimal diet was determined in two ways: (1) using a statistical criterion; and (2) computing the absolute value of the difference between the predicted energy maximized diet and observed diet (in terms of the proportion of monocot in the diet). 'Optimal' foragers were individuals whose mean observed diet was not significantly different from predicted ( $t$-test, $p>0.05$ ), given the variance among fecal pellets within a day. Individuals with observed diets significantly different from predicted were called 'deviators'. By this statistical criterion, 67 of 109 individuals foraged optimally.

The frequency distribution of the difference between predicted and observed diets is not significantly different from a normal (symmetric) distribution with the same mean and variance (Fig. 4a) (goodness-of-fit, $\chi^{2}=22.2, p>0.10, n=109$, df $=15$ ). There is no apparent tendency for 'deviators' to consume one particular food type in greater proportion than predicted.

I expressed deviations as absolute values because any deviation from optimum results in less energy intake for the forager, regardless of whether a disproportionate share of monocot or dicot is consumed. The frequency distribution of these deviations is significantly skewed to the right relative to a Poisson (random) distribution with the same mean (Fig. 4b) $\left(\chi^{2}=38.6, \mathrm{df}=7, p<\right.$ 0.001 ). As a result, both measures of deviation suggest that some individuals deviate significantly from their optimal diet.

An individual's deviation from its optimal diet does not appear to depend on its sex. Median deviation from an optimal diet does not differ between males and females for either adults (Kruskal-Wallis, $\mathrm{K}-\mathrm{W}, H=0.54, p>0.20, n=50)$ or juveniles $(\mathrm{K}-\mathrm{W}, H=5.23, p>0.15, n=$ 

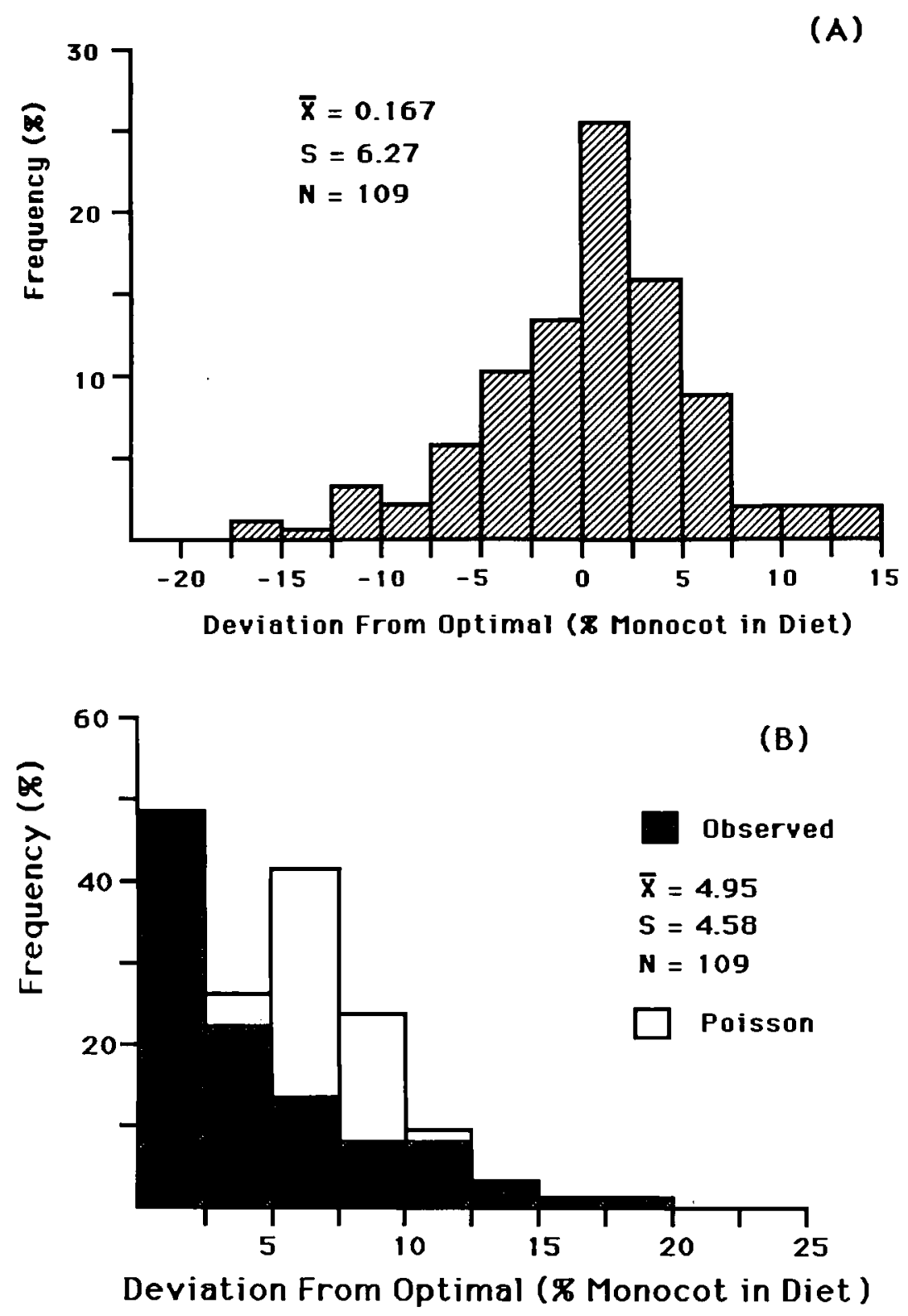

Figure 4. The frequency distributions of deviation from an optimal diet (as \% monocot in the diet) are presented. In (a), the actual difference between observed and predicted diets is shown, with positive differences signifying excess consumption of monocot. In (b), the distribution of the absolute values of deviations, my measure of foraging performance, is shown (shaded bars), along with a Poisson (random) distribution with the same mean (open bars). In each case, sample size, mean and S.D. are shown.

59). This suggests that males and females do not have different foraging strategies, at least during the non-breeding season.

To estimate the potential error in the energy maximized prediction caused by errors in 
parameter measurement, I used a Monte Carlo simulation technique to generate a mean and variance for the predicted energy maximized diet. In this study, the simulation used the following algorithm. (1) Use the observed means and standard errors to define normal distributions for the means of six model parameters that determine the energy maximized solution (feeding time, cropping rates of monocots and dicots, digestive capacity, and the bulk of monocots and dicots). (2) Take one random sample from the distribution of means for each parameter to provide a set of parameters for solving the linear programming model. (3) Solve the model for the energy maximized diet proportion. (4) Repeat this process 100 times to provide a distribution of energy maximized diet proportions. (5) Repeat steps (1)-(4) for each of the 109 individuals.

The impact of model sensitivity was calculated by comparing the mean and standard error of the predicted energy maximized diet and observed diet for each individual using two-sample $t$ tests. Among 37 individuals originally classified as deviators, 30 had observed diets significantly different from their mean energy maximized diet predicted by the Monte Carlo simulation. As a result, $82 \%$ of deviators still deviated significantly from their optimal diet when potential errors in parameter measurement were taken into account.

An individual's deviation from its optimal diet also does not appear to be affected by its immediate foraging environment (Fig. 5). Individuals' field deviations are significantly correlated with their deviations in the enclosures $\left(r^{2}=0.62, p<0.01\right)$. The slope of the regression line is not significantly different from one $(t=0.76, n=22, p>0.20)$, so there does not appear to be any improvement by deviators in an environment with abundant food. Individuals which foraged

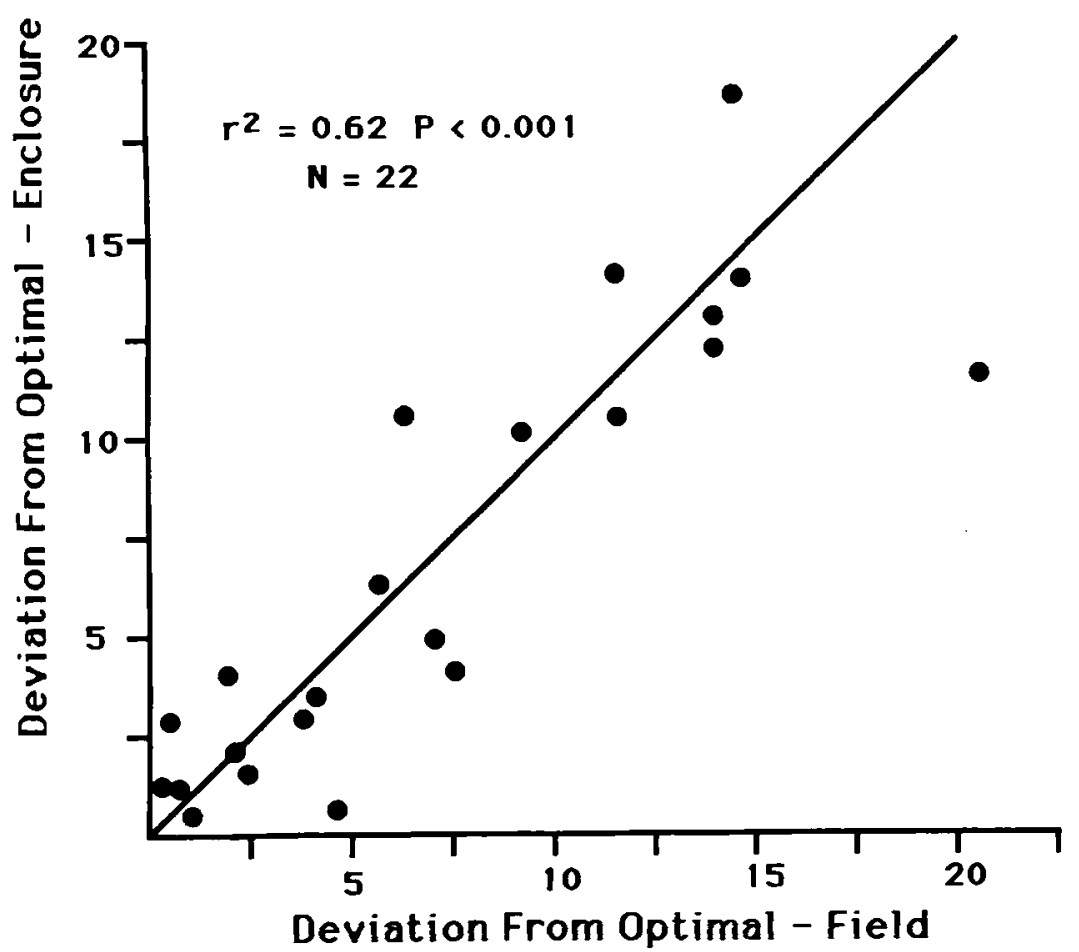

Figure 5. The correlation between deviation from an optimal diet (absolute values, $\%$ grass in the diet) of individuals in the enclosures and in the field is shown. The diagonal line represents perfect agreement. 
optimally in the field also foraged optimally in the enclosures in 10 of 10 cases, while field deviators foraged optimally in enclosures in only 2 of 12 cases (Fisher's Exact Test, $p<0.0001$ ). This indicates that individuals exhibit the same ability to select an optimal diet in a new environment. Individuals, therefore, appear to make the same number of foraging mistakes regardless of their foraging environment. This suggests that the immediate foraging environment has little influence on an individual's ability to select an optimal diet.

\section{Discussion}

All individuals appear to approach an energy maximized diet proportion, as opposed to a time minimized or random diet proportion. Some individuals, however, deviate significantly from their optimal diet. The potential importance of such variation has been largely ignored in previous studies (Pyke, 1984; Stephens and Krebs, 1986).

\section{Deviation as an individual trait}

Individuals may exhibit a particular deviation from an optimal diet, i.e. their deviation can be viewed as a behavioral trait, similar to the way in which body size can be treated as a morphological trait. An individual's deviation can be viewed as a behavioral trait if it is unmodified by the immediate foraging environment and consistent over time within a season. If deviation from an optimal diet can be viewed as an individual trait, then two consequences may follow. (1) Individuals with greater deviations from their optimal diet would lose potential daily energy intake. This loss in energy intake might be sufficient for optimal foragers to have a fitness advantage over deviators (see Ritchie, submitted) and to be favored by natural selection. (2) The appropriate trait would appear to be an individual's ability to select its own optimal diet, given its immediate foraging environment. This means that individuals would not be 'hard-wired' to select one particular diet at all times (e.g. the optimal diet for average conditions).

Deviation from an optimal diet can be viewed as an individual trait only if: (1) deviation is not the result of my sampling error in determining the predicted diet; (2) an individuals' deviation from an optimal diet is consistent over time; and (3) an individual's deviation is the same regardless of its foraging environment.

Precision of estimating deviation. My estimate of the quantitative difference between predicted and observed diets may be subject to potential sampling error. First, observed diets may have been collected before individuals had completed their foraging bout, i.e. had not had time to switch from a patch of one food type to a patch of another. Individuals were observed to switch food patches an average of every 4.3 ( \pm 3.4 S.D.) minutes, however, and all captured squirrels had fed for at least one hour before capture. Deviation from an optimal diet, therefore, should not result from interrupting foraging bouts to sample observed diets.

Error may also result because the categories of 'optimal' and 'deviator' were determined using $t$-tests based on variation among fecal pellets collected in a single day. I could not use independent diets collected on different days for every individual because many individuals could only be captured once. I obtained diets, however, on at least three separate days for 10 individuals. For each of these 10 individuals, the mean and variance were compared for diet samples collected on different days and within a day. The means for diets measured on different days were not significantly different from those measured on the same day (paired $t$-test, $t=1.65$, $\mathrm{df}=9, p>0.20$ ), and the same was true for variances (paired $F$-test, $F=1.01$, $\mathrm{df}=9, p>0.20$ ). My categories of 'optimal' vs. 'deviator', therefore, would not have changed if I had used variation in diet between days. 
A third potential source of error in quantifying deviation from an optimal diet results from the sensitivity of individual linear programming models to errors in parameter measurement. The Monte Carlo simulation provides a standard error for the predicted diet caused by errors in measuring the model parameters. If this standard error is included in the statistical comparisons of predicted and observed diets, only 7 of 109 individuals would be reclassified as 'optimal' or 'deviating' foragers. This suggests that the observed variances in parameter measurements have little impact on the conclusions of this study.

I chose not to classify foraging ability based on these simulated standard errors because: (1) they are artificial and depend on how many calculations of the predicted diet are made in the simulation (e.g. 100 in this study); and (2) the difference between the average predicted and observed diets for an individual still provides the best information about the individual's success in selecting its optimal diet.

Consistency in deviation. Is an individual's deviation from an optimal diet consistent over time? The results of the enclosure experiments suggest that an individual's deviation from an optimal diet is consistent over 4-6 weeks (approximately $50 \%$ of the active season). This conclusion arises because the enclosure experiments represent an independent determination of an individual's deviation from its optimal diet after it was measured in the field.

Deviation from an optimal diet was also compared between years for individuals observed in consecutive years. Although individuals are consistent in their deviation within a season, they appear to improve from one year to the next. Deviation in year 2 was significantly correlated with deviation in the previous year $\left(r^{2}=0.83, p<0.001, n=11 ; \mathrm{y}=0.65( \pm 0.08 \mathrm{SE}) \mathrm{x}+0.56\right)$. The slope of this equation is significantly less than one $(t=4.31, p<0.001)$, indicating significant improvement between years.

Ground squirrels, therefore, appear to be very consistent in their foraging behavior within the active season, although they show some ontogenetic improvement between years. This allows fairly precise estimates of individuals' deviations from their optimal diet within a season $( \pm 20 \%$ of the estimate) to be obtained from a few days of measurements.

Environmental influences. Does an individual's deviation from an optimal diet vary with the immediate foraging environment? I define the immediate foraging environment to be the combination of factors, e.g. thermal conditions, food abundance, social interactions, which potentially affects food consumption by ground squirrels. The enclosures provide a completely new foraging environment for each individual.

The results of the enclosure experiments (Fig. 5) suggest that: (1) an individual's deviation from its optimal diet does not change with its immediate foraging environment; and (2) individuals are not more likely to improve their deviation than expected by chance $\left(\chi^{2}=0.36\right.$, $\mathrm{df}$ $=1, p>0.20, n=22$ ). Individuals do not deviate consistently in favor of one food type or the other since the quantity (observed diet - predicted diet) is not correlated between field and enclosure $\left(r^{2}=0.04, p>0.20\right)$.

The magnitude of deviation, however, is consistent between field and environment. For example, an individual, which ate $35 \%$ monocot when its optimal diet in the field was $30 \%$, ate $15 \%$ monocot in the enclosures when its optimal diet was $20 \%$. Its deviation from an optimal diet in both cases was 5\%. This suggests that an individual's deviation from its optimal diet in favor of monocots or dicots is independent of the absolute magnitude of its deviation.

For this example, the change in observed diet between environments is greater than the change in predicted optimal diets. The error in diet selection, however, is the behavior of interest, since this reflects the individual's energy intake relative to other individuals and potentially its relative 
fitness in the population. Consequently, it is only important to measure the effect of the environment on an individual's deviation from its optimal diet, rather than on its observed diet.

Individuals might deviate in the direction of their random diet, since incorrect choices of food items might occur in proportion to the relative abundance of the two food types in the environment. For example, individuals with optimal diets containing a lower proportion of dicots than found in the environment might deviate from their optimal diet in favor of dicots. This is not supported, however, since individuals with optimal diets containing a lower proportion of dicots than in the environment did not deviate in favor of dicots more often than expected by chance $(50 \%)\left(\chi^{2}=1.27\right.$, df $\left.=1, p>0.20, n=109\right)$.

The predicted energy maximized diets for individuals in the enclosures are not correlated with their corresponding predicted diets in the field $\left(r^{2}=0.001, n=22, p>0.90\right)$. This result suggests that individuals were apparently able to adjust their foraging behavior appropriately to an unforeseen event. Ground squirrels, consequently, may learn the distribution of plants in their environment and select a diet that maximizes their daily energy intake (Slobodkin and Rapoport, 1974; Shettleworth, 1984). As a result, individuals do not appear to be 'hard-wired' to choose a particular diet regardless of conditions. The important character of foraging behavior, rather, may be an individual's ability to track environmental changes and to determine the optimal diet for the current conditions. Deviators, therefore, may be unable to correctly decide what balance of monocots and dicots to select in a given environment.

The above results suggest that deviation from an optimal diet is intrinsic to individual ground squirrels, i.e. the deviation of an individual is predictable in time and unaffected by the immediate environment. This notion has important consequences for studying optimal foraging in populations.

\section{The advantage of optimal foraging behavior}

If deviation from optimal is intrinsic to an individual, then it can be viewed as a trait which might be subject to natural selection (Endler, 1986). Selection against deviators in the population can be demonstrated by comparing median deviations of different age classes (Fig. 6). Median adult deviation from optimal was less than that for juveniles (K-W, $H=30.5, p<0.001, n=109$ ).

There are two potential explanations for this observation: (1) ontogenetic improvement in deviation from an optimal diet; and (2) higher mortality of juvenile deviators, thereby reducing the median deviation of older classes. Ontogenetic improvement does occur (see earlier), but the shift in median deviation may also result from mortality of deviating juveniles.

For optimal foraging to be advantageous to an individual, deviation from an optimal diet must result in a significant reduction in the 'currency' for fitness. This 'currency' appears to be energy (Ritchie, submitted). The loss in potential energy intake due to deviation from an optimal diet is only important if deviators have the same activity time, cropping rates, and digestive capacity, assimilation and conversion efficiency of food, etc., as optimal foragers. This also means that optimal and deviator foragers must be equal in body size, since larger individuals would have greater energy intake, on average, due to their greater digestive capacity. Body size and the linear programming model parameters do not differ among optimal and deviator foragers of a given sex-age class (Ritchie, submitted). Experiments with captive individuals also show that optimal and deviating foragers do not differ in their assimilation and conversion efficiency (Ritchie, submitted). Differences in energy intake among optimal and deviating foragers, therefore, arise only from differences in their deviation from an optimal diet.

The lost potential energy intake suffered by deviators appears to be sufficient to reduce their fitness (Ritchie, submitted). Optimal foragers have faster growth rates, greater survivorship, and larger litters. This performance leads to a large $(100 \times)$ fitness advantage for optimal foragers 


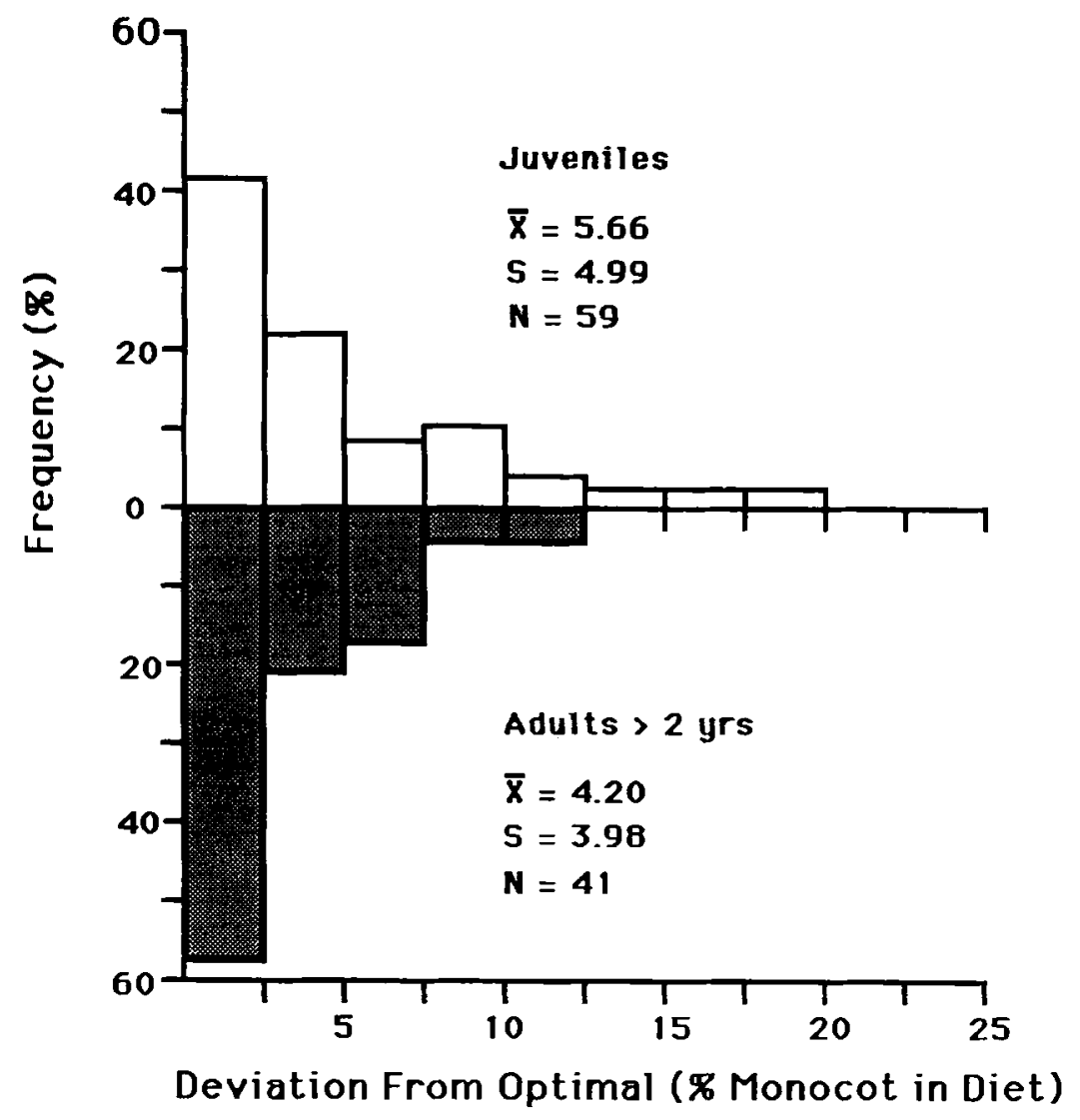

Figure 6. The distributions of deviations (absolute values, \% grass in the diet) of adults (shaded bars) and juveniles (open bars) are compared. Sample size, mean and S.D. are provided in each case.

from birth to age three. These large fitness differences are not surprising, since this species has a short active season (110 days at my study site) to grow, reproduce, and store sufficient fat to survive 255 days of hibernation/estivation. Consequently, any small difference between individuals in daily energy intake will be compounded during the accumulation of fat for hibernation and production of offspring.

Ritchie (submitted) also shows that juvenile 'deviators' are more likely to die than 'optimal' juveniles. This suggests that the smaller average deviation of adults (Fig. 6) results in part from the disappearance of 'deviating' individuals from a particular cohort over time. Both ontogenetic improvement and mortality of juveniles appear to contribute to the improvement in foraging ability with age observed in the population.

If natural selection favors optimal foraging behavior, it is likely to favor the ability to choose an optimal diet. Individuals appear to adjust their diet with changing conditions, even though their deviation from their optimal diet does not change over the season. In a changing environment, individuals that can adjust their diet to meet environmental conditions would have a greater energy intake over the season than individuals that eat a single diet that is optimal for average conditions. Foraging conditions for ground squirrels often change substantially due to yearly variations in the thermal environment, cattle grazing schedules, rainfall, etc. (Morton and Sherman, 1978; Boag and Murie, 1981b; Dobson and Kjelgaard, 1985; Belovsky and Ritchie, 
1989). It is therefore not surprising that ground squirrels are highly plastic in their diet choice (Fig. 3a).

\section{Conclusion}

Individual ground squirrels appear to vary in their ability to achieve the diet that maximizes their daily energy intake, as $37 \%$ of individuals deviated significantly from their optimal diets. Deviation from an optimal diet appears to be intrinsic to an individual, i.e. does not vary within a season or with foraging environment. Deviation appears to arise because individuals are unable to correctly determine their optimal diet proportion for a given environment.

These results have potentially important consequences for studying optimal foraging in populations. First, deviation from an optimal diet can be viewed as an individual behavioral trait. Second, deviators suffer energy losses from their incorrect diet choice, which are sufficient to reduce their fitness. Finally, if natural selection favors optimal foraging behavior, the ability to choose the optimal diet for different environmental conditions is probably the trait selected rather than a 'hard-wired' preference for a particular diet.

\section{Acknowledgments}

I thank G. E. Belovsky, J. S. Diana, D. I. Rubenstein, O. J. Schmitz, P. E. Smouse, P. W. Webb and an anonymous reviewer for critically reading various drafts of this manuscript. I thank $J$. Brady, L. Evans, R. Jensen and W. Pearson for access to their land near the National Bison Range. I also thank my field assistants R. Henderson, D. McCullough, J. Richter and S. Wall. The work was supported by NSF grant BSR-8307352 to G. E. Belovsky, a Doctoral Dissertation Improvement grant BSR-8501040 to G. E. Belovsky and M. E. Ritchie, Grants-in-Aid from Sigma Xi, the Scientific Research Society, in 1983 and 1985, and a Rackham Predoctoral Fellowship from the University of Michigan.

\section{References}

Arnold, S. J. (1977) Polymorphism and geographic variation in the feeding behavior of the garter snake, Thamnophis elegans. Science 197, 676-8.

Arnold, S. J. (1981) The microevolution of feeding behavior. In Foraging Behavior: Ecological, Ethological, and Psychological Approaches (A. C. Kamil and J. D. Sargent, eds.). Garland Press, NY, USA.

Belovsky, G. E. (1978) Diet optimization in a generalist herbivore: the moose. Theor. Pop. Biol. 14, 105-34.

Belovsky, G. E. (1984) Herbivore optimal foraging: a comparative test of three models. Amer. Natur. 124, 97-115.

Belovsky, G. E. (1986) Optimal foraging and community structure: implications for a guild of generalist grassland herbivores. Oecologia 70, 35-62.

Belovsky, G. E. and Ritchie, M. E. (1989) Geographical and seasonal variation in foraging of Columbian ground squirrels. Oecologia (in press).

Belovsky, G. E. and Slade, J. B. (1986) Time budgets of grassland herbivores: body size similarities. Oecologia 70, 53-62.

Boag, D. A. and Murie, J. O. (1981a) Weight in relation to sex, age, and season in Columbian ground squirrels (Sciuridae: Rodentia). Canad. J. Zool. 59, 999-1004.

Boag, D. A. and Murie, J. O. (1981b) Population ecology of Columbian ground squirrels in southwestern Alberta. Canad. J. Zool. 59, 2230-40.

Charnov, E. L. (1976) Optimal foraging: attack strategy of a mantid. Amer. Natur. 110, 141-51. 
Dobson, F. S. and Kjelgaard, J. D. (1985) The influence of food resources on population dynamics in Columbian ground squirrels. Canad. J. Zool. 63: 2105-9.

Emlen, J. M. (1966) The role of time and energy in food preference. Amer. Natur. 100, 611-17.

Endler, J. A. (1986) Natural Selection in the Wild. Princeton University Press, Princeton, NJ, USA.

Hansen, R. M., Foppe, T. M., Gilbert, M. B., Clark, R. C. and Reynolds, H. W. (1977) The Microhistological Analysis of Feces as an Estimator of Herbivore Diet. Composition Analysis Lab, Colorado State University, Ft Collins, USA.

Hixon, M. A. (1982) Energy maximizers and time minimizers: theory and reality. Amer. Natur. 119, 596-9.

Houston, A. I., Krebs, J. R. and Erichsen, J. T. (1980) Optimal prey choice and discrimination time in the great tit (Parus major L.). Behav. Ecol. Sociobiol. 6, 169-75.

Hudson, J. W. and Deavers, D. R. (1973) Metabolism, pulmo-cutaneous water loss and respiration of eight species of ground squirrels from different environments. Comp. Biochem. Physiol. (A) 45, 69-100.

Intriligator, M. D. (1971) Mathematical Optimization and Economic Theory. Columbia Press, NY, USA.

Karasov, W. H. (1981) Daily energy expenditure and the cost of activity in a free-living mammal. Oecologia 51, 253-9.

Kilgore, D. L. Jr and Armitage, K. B. (1978) Energetics of yellow-bellied marmot populations. Ecology 59, 78-88.

Kleiber, M. (1961) The Fire of Life. Wiley Press, NY, USA.

Krebs, J. R., Ryan, J. C. and Charnov, E. L. (1974) Hunting by expectation or optimal foraging? A study in patch use by chickadees. Anim. Behav. 22, 953-64.

Krebs, J. R., Erichsen, J. T., Webber, M. I. and Charnov, E. L. (1977) Optimal prey selection in the Great Tit (Parus major). Anim. Behav. 25, 30-8.

MacArthur, R. H. and Pianka, E. R. (1966) On optimal use of a patchy environment. Amer. Natur. 100, $603-9$.

Manville, R. H. (1959) The Columbian ground squirrel in northwestern Montana. J. Mamm. 40, $26-45$.

Morhardt, S. S. (1975) Use of climate diagrams to describe microhabitats occupied by Belding ground squirrels and to predict rates of change of body temperature. In Perspectives in Biophysical Ecology (D. M. Gates and R. B. Schmerl, eds), pp. 303-23. Springer-Verlag, Berlin.

Morhardt, S. S. and Gates, D. M. (1974) Energy exchange analysis of the Belding ground squirrel and its habitat. Ecol. Monog. 44, 17-44.

Morton, M. L. and Sherman, P. W. (1978) Effects of a spring snowstorm on behavior, reproduction, and survival of Belding's ground squirrels. Canad. J. Zool. 56, 2578-90.

Porter, W. P. and Gates, D. M. (1969) Thermodynamic equilibria of animals with environment. Ecol. Monog. 39, 227-44.

Pulliam, H. R. (1974) On the theory of optimal diets. Amer. Natur. 108, 59-74.

Pulliam, H. R. (1975) Diet optimization with nutrient constraints. Amer. Natur. 109, 765-8.

Pyke, G. H. (1984) Optimal foraging: a critical review. Ann. Rev. Ecol. Syst. 15, 523-75.

Pyke, G. H., Pulliam, H. R. and Charnov, E. L. (1977) Optimal foraging: a selective review of theory and tests. Q. Rev. Biol. 52, 137-54.

Rechten, C., Avery, M. I. and Stevens, T. A. (1983) Optimal prey selection: why do great tits show partial preferences? Anim. Behav. 31, 576-84.

Ritchie, M. E. Optimal foraging and fitness in Columbian ground squirrels. (submitted).

Schitoskey, F. and Woodmansee, S. R. (1978) Energy requirements and the diet of the California ground squirrel. J. Wildlife Mgemnt. 42, 373-82.

Schoener, T. W. (1971) Theory of feeding strategies. Ann. Rev. Ecol. Syst. 2, 369-403.

Shaw, W. T. (1925) The food of ground squirrels. Amer. Natur. 59, $250-64$.

Shettleworth, S. J. (1984) Learning and behavioural ecology. In Behavioural Ecology: An Evolutionary Approach 2nd edn. (J. R. Krebs and N. B. Davies, eds), pp. 170-94. Sinauer Associates, Sunderland, MA, USA.

Slobodkin, L. B. and Rapoport, A. (1974) An optimal strategy of evolution. Q. Rev. Biol. 49, 181-200.

Sparks, D. R. and Malechek, J. C. (1968) Estimating percentage dry weight in diets using a microscopic technique. J. Range Mgemnt. 21, 264-5. 
Stephens, D. W. and Krebs, J. R. (1986) Foraging Theory. Princeton University Press, Princeton, NJ, USA.

Storr, G. M. (1963) Estimation of dry matter intake in wild herbivores. Nature 197, 307-8.

Wang, L. C. H. (1979) Time patterns and metabolic rates of natural torpor in the Richardson's ground squirrel. Canad. J. Zool. 57, 149-55.

Webb, D. R. (1980) Environmental harshness, heat stress, and Marmota flaviventris. Oecologia 44, 390-5.

Werner, E. E. and Hall, D. J. (1974) Optimal foraging and the size selection of prey by the bluegill sunfish (Lepomis macrochirus). Ecology 55, 1042-52.

Westoby, M. (1974) An analysis of diet selection by large generalist herbivores. Amer. Natur. 108, $209-304$. 